\title{
Artroplastía Total de Rodilla con Asistencia de Brazo-Robótico: Resultados Clínicos Postoperatorios Tempranos
}

\section{Robotic Arm-Assisted Total Knee Arthroplasty: Early Postoperative Results}

\author{
David Figueroa ${ }^{1}$ Rodrigo Guiloff ${ }^{1} \quad$ Marcela Naranjo $^{1} \quad$ María Loreto Figueroa $^{2} \quad$ Alex Vaisman $^{1}$ \\ Carlos Stierling ${ }^{1}$ Rafael Calvo ${ }^{1}$ \\ ${ }^{1}$ Facultad de Medicina Clínica Alemana, Universidad del Desarrollo, \\ Santiago, Chile \\ 2 Facultad Medicina, Pontificia Universidad Católica de Chile, Chile \\ Address for correspondence Rodrigo Guiloff, MD, Facultad de \\ Medicina Clínica Alemana-Universidad del Desarrollo, Santiago, \\ Chile (e-mail: rguiloff@gmail.com).
}

Rev Chil Ortop Traumatol 2019;60:97-105.

\begin{abstract}
Resumen
Palabras claves

- gonartoris

- artroplastía total de rodilla

- asistencia robótica

Objetivo Describir los resultados clínicos postoperatorios tempranos de la primera serie de pacientes operados de artroplastía total de rodilla (ATR) con asistencia de brazo-robótico en Latinoamérica.

Materiales y Métodos Estudio prospectivo de 52 pacientes (53 rodillas) con gonartoris tricompartimental sintomática operados de ATR primaria con asistencia de brazo-robótico (RIO-MAKO) de manera consecutiva, con seguimiento a 2 meses postoperados. No se excluyeron pacientes. Se utilizó el mismo protocolo anestésico y de rehabilitación. Se realizó revisión de fichas clínicas para recolectar los siguientes resultados: tiempo de isquemia quirúrgica, estadía hospitalaria, tiempo a marcha, dolor postoperatorio diario [Escala visual análoga (EVA)], uso de opioides, rangos de movilidad articular (ROM), pérdida sanguínea, complicaciones y eje mecánico postoperatorio.

Resultados Tiempos de Isquemia: 82 minutos (60-120). Estadía Hospitalaria: 4 días (2-12). Dolor postoperatorio: EVA el mismo día operatorio de $0(0-10)$ y previa al alta de 0 (rango $0-4)$, con 0,3 purgas $(0-6,5)$ de opioides por paciente. Tiempo a marcha (día logrado): 1 día (1-3), cuarenta y cinco pacientes (84,9\%) iniciaron la marcha el primer día postoperatorio. ROM (Extensión-Flexión): Aumento progresivo durante el seguimiento. En el control ambulatorio de los dos primeros meses [23 días (13-50)], veintiún pacientes $(40 \%)$ alcanzaron un $\mathrm{ROM}$ mayor o igual a $0-90^{\circ}$ y once $(20 \%)$ presentaron un ROM funcional máximo $\left(0-120^{\circ}\right)$. Pérdida sanguínea: Siete pacientes $(13,2 \%)$ requirieron transfusión. Complicaciones: un paciente $(1,89 \%)$ presentó una dehiscencia del cierre de la artrotomía. No hubo otras complicaciones. Eje mecánico postoperatorio: $179,1^{\circ}(178,2-180)$.

Discusión Los resultados concuerdan con la evidencia descrita en otras regiones, sugiriendo que la asistencia de brazo-robótico permite resultados postquirúrgicos reproducibles.
\end{abstract}

received

July 12, 2019

accepted

September 24, 2019
DOI https://doi.org/

10.1055/s-0039-3399544. ISSN 0716-4548.
Copyright (e 2019 by Thieme Revinter

Publicações Ltda, Rio de Janeiro, Brazil
License terms

(요 (1) $\Theta$ 


\begin{abstract}
Keywords

- knee osteoarthritis

- total knee replacement

- robotic assistance

Objective Describe the early clinical postoperative outcomes, of the first series of patients operated on Robotic Arm-Assisted Total Knee Arthroplasty (TKA) in Latin America.

Material and Method Retrospective study including 52 patients (53 knees) with advanced symptomatic knee osteoarthritis, consecutively operated on Robotic ArmAssisted TKA (RIO-MAKO) during October 2018 and May 2019 with two months of follow-up. No patients were excluded from the study. The same anesthetic and rehabilitation protocol was followed for all patients. Data were obtained from the clinical files for the following outcomes: Surgical tourniquet time, hospital stay, time to walk, postoperative daily pain [Visual Analog Scale (VAS)], opioid consumption, range of motion (ROM), blood loss, complications, and the attained postoperative mechanical axis.

Results Tourniquet Time: 82 minutes (60-120). Hospital Stay: 4 days (2-12). Postoperative Pain: VAS of $0(0-10)$ the same day of surgery, and $0(0-4)$ before discharge. The opioid consumption was 0,3 purge $(0-6,5)$ per patient. Time to Walk (Day achieved): $1^{\text {st }}$ day (1-3), forty-five patients (84,9\%) walked on their first postoperative day. ROM (Extension-Flexion): progressively increases during the follow up. On the first two-month visits [23 days (13-50)], twenty-one patients (40\%) reached a ROM equal or superior to $0-90^{\circ}$, and eleven $(20 \%)$ presented a maximal functional ROM $\left(0-120^{\circ}\right)$. Blood Loss: Seven patients $(13.2 \%)$ required a blood transfusion. Complications: one patient (1.89\%) presented dehiscence of the arthrotomy closure. No other complications were registered. Postoperative Mechanical Axis: $179.1^{\circ}$ (178.2-180).

Discussion The results coincide with the reported evidence from other regions. Robotic-arm assistance may generate reproducible postoperative results.

Conclusions The early postoperative clinical results of this series of patients operated on Robotic Arm-Assisted TKA show a low pain perception and opioid use, a rapid functional rehabilitation in terms of gait and ROM, and excellent postoperative mechanical alignment.

Level of Evidence IV.
\end{abstract}

Conclusión Los resultados clínicos postoperatorios tempranos de esta serie de pacientes operados de ATR con asistencia de brazo-robótico, muestran una baja percepción del dolor, con bajo consumo de opioides, una rápida recuperación funcional de la marcha y ROM, y excelentes resultados desde el punto de vista del eje mecánico postoperatorio.

Nivel de Evidencia IV.

\section{Introducción}

La gonartrosis (GA) es la forma más frecuente de artrosis, su prevalencia global se ha duplicado en el último siglo y sigue en aumento. ${ }^{1}$ Es considerada una de las causas más comunes de incapacidad permanente en mayores de 65 años. ${ }^{2,3}$ En etapas terminales de la enfermedad, la artroplastía total de rodilla (ATR) es considerada el estándar terapéutico, realizándose en Estados Unidos anualmente más de 700.000 procedimientos, con una proyección esperada para la próxima década en 3 millones de ATR/año. ${ }^{4}$

A pesar de los avances en investigación, diseño de prótesis, técnicas quirúrgicas y rehabilitación postoperatoria, existe una proporción variable cercana al $20 \%$ de insatisfacción posterior a una ATR y una tasa de revisión protésica que alcanza entre un $5,4 \%$ a $18 \%$ entre los 8 y los 25 años respectivamente. ${ }^{5,6} \mathrm{La}$ inestabilidad protésica es responsable de un 7,3 a $28,9 \%$ de las revisiones, que puede ser consecuencia de un mal posicionamiento de los componentes protésicos durante la cirugía. $5,7,8$

Con el objetivo de prevenir este problema, han aparecido la navegación y los sistemas robóticos. ${ }^{9,10}$ Específicamente en la cirugía protésica de rodilla esas técnicas han demostrado aumentar la exactitud de los cortes óseos, la precisión del posicionamiento del implante y una mayor protección de los 
tejidos blandos, con un aumento potencial de la seguridad de ese procedimiento. ${ }^{10-13}$ Sin embargo, la repercusión clínica en términos de mejora en la tasa de complicaciones y sobrevida del implante con nuevas revisiones no ha sido demostrada. $^{10-14}$

Estudios comparativos recientes entre la cirugía protésica de rodilla con asistencia robótica y la cirugía convencional han evidenciado diferencias significativas en el postoperatorio inmediato. La asistencia robótica ha permitido un postoperatorio con menor dolor y menor pérdida sanguínea, mayor funcionalidad física, una rehabilitación precoz del rango de movilidad articular (ROM), mejor percepción de salud y mayor satisfacción general. ${ }^{11,12,14}$ Sin embargo, la literatura es limitada y poco representativa, pues proviene en su mayoría de países anglosajones y asiáticos. ${ }^{11-17}$ A nuestro entender, no existen reportes clínicos en Latinoamérica sobre ese tema.

El objetivo de este trabajo es describir los resultados clínicos tempranos incluyendo el postoperatorio inmediato, de una serie de pacientes operados de ATR asistida por brazo-robótico en un centro de salud Latinoamericano.

\section{Materiales y Métodos}

\section{Diseño de la Investigación}

Estudio descriptivo, prospectivo.

\section{Selección de Pacientes}

Se incluyeron 52 pacientes (53 rodillas) con artrosis de rodilla sintomática que se sometieron a ATR con asistencia de brazo-robótico de manera consecutiva entre octubre de 2018 y mayo de 2019. Los procedimientos fueron realizados por 3 cirujanos de rodilla, de un mismo centro, todos capacitados en reemplazo articular de rodilla con asistencia de brazo-robótico.

Los criterios de inclusión fueron: pacientes con artrosis tricompartimental avanzada de rodilla (KellgrenLawrence $\geq 3$ ) sintomática con indicación de ATR por su médico tratante, cirugía de ATR primaria, operados con asistencia de brazo-robótico y con consentimiento informado por escrito. No se excluyeron pacientes del estudio.

Los datos demográficos de los pacientes incluidos se resumen en la - Tabla $\mathbf{1}$. Un paciente recibió una ATR bilateral en el mismo procedimiento, se consideró cada rodilla de forma individualizada.

\section{Materiales Utilizados}

Se realizó una revisión de fichas clínicas y una encuesta telefónica a los pacientes incluidos en el estudio.

\section{Técnica Quirúrgica}

\section{Insumos}

Los procedimientos quirúrgicos fueron realizados con asistencia del mismo brazo-robótico: Robotic Arm Interactive Orthopedic System (RIO) (MAKO Surgical Corp., Stryker®, Fort Lauderdale, FL, USA). Ese sistema cuenta con un sensor de registro espacial que proyecta una imagen tridimensional (3D)
Tabla 1 Demografía de pacientes operados de ATR con asistencia de brazo-robótico ( $n=53$ rodillas)

\begin{tabular}{|c|c|}
\hline Categoría & \\
\hline Edad Mediana (Rango) & $71(53-86)$ \\
\hline \multicolumn{2}{|l|}{ Género, n (\%) } \\
\hline Femenino & $37(71,2 \%)$ \\
\hline Masculino & $16(30,8 \%)$ \\
\hline IMC (kg/m2) Mediana (Rango) & $27,9(20-45,3)$ \\
\hline \multicolumn{2}{|l|}{ Comorbilidades, n (\%) } \\
\hline HTA & $24(45,3)$ \\
\hline DM & $9(17,3)$ \\
\hline Hipotiroidismo & $10(18,9)$ \\
\hline Cardiopatía & $3(5,7)$ \\
\hline ACV & $1(1,9)$ \\
\hline Parkinson & $1(1,9)$ \\
\hline Fibromialgia & $2(3,8)$ \\
\hline Cáncer & $2(3,8)$ \\
\hline Depresión & $16(30,2)$ \\
\hline Tabaco, n (\%) & $8(15,1)$ \\
\hline \multicolumn{2}{|l|}{ ASA (grado), n (\%) } \\
\hline 1 & $9(17,0)$ \\
\hline II & $42(79,2)$ \\
\hline III & $2(3,8)$ \\
\hline IV & $0(0)$ \\
\hline $\begin{array}{l}\left.\text { ROM Preop (Ex/Flex }{ }^{\circ}\right) \\
\text { Mediana (Rango) }\end{array}$ & $0 / 125(0 / 100-20 / 140)$ \\
\hline \multicolumn{2}{|l|}{ Lateralidad de intervención, n (\%) } \\
\hline Unilateral & $51(96,2)$ \\
\hline Bilateral & $2(3,8)$ \\
\hline
\end{tabular}

Abreviaturas: ACV, Accidente Cerebro Vascular; DM, Diabetes Mellitus; Ex, Extensión; Flex, Flexión; HTA, Hipertensión Arterial; IMC, Índice de Masa Corporal; Preop, Preoperatorio; ROM, Rango de movilidad articular.

en una pantalla durante la cirugía. La patela fue reemplazada en todas las cirugías y se utilizó el mismo implante protésico en todos los pacientes: Triathlon PS (Stryker ${ }^{\circledR}$, Fort Lauderdale, FL, USA).

\section{Protocolo Anestésico}

Se consideró el mismo protocolo anestésico: anestesia general e infiltración local de la cápsula posterior y espacios medial y lateral durante la cirugía, con una combinación de chirocaína, dexmedetomidina y ketorolaco, previo a la colocación de los componentes. Se administró $1 \mathrm{~g}$ de ácido tranexámico al inicio de la cirugía y al momento de soltar la isquemia.

\section{Planificación Preoperatoria}

La cirugía fue planificada utilizando una tomografía computarizada (TC) preoperatoria de cada paciente con un protocolo específico para el sistema RIO-MAKO que permitió el diseño de un modelo 3D de la rodilla a operar. Con ese sistema, 
se establecieron los tamaños y el posicionamiento de los implantes en base a los ejes mecánicos del plano coronal, sagital y transversal, la inclinación tibial posterior nativa, el ángulo de flexión-extensión y la rotación interna-externa de la rodilla.

\section{Protocolo Quirúrgico}

Todos los pacientes fueron posicionados en decúbito supino. Se instaló un manguito de isquemia en la extremidad a ser operada, el que fue inflado posterior a la colocación de los campos quirúrgicos de las cirugías de dos cirujanos (un cirujano operó sin isquemia). Se utilizó un abordaje parapatelar medial en todos los procedimientos, con mínima liberación de tejidos blandos a medial. Alcanzado el tejido óseo, se colocaron los trackers de registro femoral y tibial y se corroboró la correcta posición de ambos en la pantalla de registro. A continuación, se resecaron los osteofitos y se procedió a la corroboración de la planificación preoperatoria con pruebas dinámicas, buscando un balance de partes blandas adecuado en extensión y flexión. Ese proceso es registrado y grabado en el sistema computarizado para realizar posteriormente los cortes óseos. Conforme con la planificación, se procedió a realizar los cortes óseos con el sistema RIO-MAKO, según los puntos de referencia previamente planificado. El sistema robótico utiliza una sierra oscilante unida a su brazo y ese es controlado por los cirujanos con retroalimentación háptica, visual y acústica para mantener los límites estereostáticos fijados por el computador. Realizado ese proceso, se probaron los implantes y se verificó nuevamente el balance de partes blandas adecuado en extensión y flexión, buscando un eje mecánico de $180^{\circ}$, y estabilidad medio lateral. Satisfechos con la prueba, se procedió a cementar los componentes definitivos. La cirugía finalizó con una hemostasia adecuada tras soltar el manguito de isquemia posterior a la cementación y un cierre por planos.

\section{Cuidados Postoperatorios}

Todos los pacientes recibieron un protocolo de analgesia postoperatoria con una bomba de analgesia controlada por el paciente (PCA) de morfina endovenosa, programada a 0-1-8, es decir, sin infusión continua y bolos de $1 \mathrm{mg}$ a demanda del paciente espaciados por al menos 8 minutos, la cual se mantuvo hasta lograr la deambulación con menos de 4 purgas en 24 horas. Adicionalmente se administró de forma horaria pregabalina y tramadol de liberación prolongada por vía oral, paracetamol y dos anti-inflamatorios no esteroidales (AINEs) de diferentes vías de acción, inicialmente por vía endovenosa y luego por vía oral al tercer día del postoperatorio en caso de presentar un dolor bien tolerado. La evaluación del dolor se realizó mediante la escala visual análoga (EVA) y se registró a diario el número de purgas de PCA.

Todos los pacientes siguieron un programa de rehabilitación postoperatorio estandarizado, con frío local intermitente, movilizador pasivo continuo por 2 horas al menos 2 veces al día desde la llegada a la sala de recuperación postquirúrgica, ejercicios de movilidad articular activa y carga a tolerancia con 2 bastones desde el día de la cirugía. Se realizaron 2 sesiones de fisioterapia al día durante toda la hospitalización. Cada sesión tuvo una duración de 20 a 30 minutos y fueron realizadas por el mismo equipo kinésico.

Los pacientes fueron dados de alta al lograr un adecuado manejo del dolor con analgesia oral, buena tolerancia a la marcha asistida con 2 bastones y una flexión de rodilla mayor o igual a $60^{\circ}$.

\section{Variables a Medir}

Se analizaron las siguientes variables: tiempo de isquemia quirúrgica; días de hospitalización; tiempo a marcha asistida (día de hospitalización en que fue lograda, se consideró el día quirúrgico como Día 0); dolor (EVA) postoperatorio diario (valoración durante la mañana y noche) hasta el alta; uso de opioides (número de purgas de PCA diaria por paciente); ROM (medido por kinesiólogo con el uso de un movilizador pasivo continuo); ROM (medido por traumatólogo con goniómetro estándar) el primer día postoperatorio y en el control ambulatorio de los dos primeros meses; pérdida sanguínea intraoperatoria (estimada por la diferencia del hematocrito previo y posterior a la cirugía y el requerimiento de transfusión, que se consideró en todo paciente con Hematocrito menor a $25 \%$ sintomático); complicaciones inmediatas y agudas tales como infección o dehiscencia de herida operatoria, enfermedad tromboembólica (trombosis venosa profunda, tromboembolismo pulmonar y embolia grasa) e infección periprotésica; y el eje mecánico final postoperatorio de la extremidad inferior (Telemetría de extremidades inferiores).

\section{Análisis Estadístico}

Se utilizó la correlación de Spearman para buscar asociaciones entre variables $(p=0,05)$. El análisis de datos, fue realizado en SPSS software versión 22.0 (SPSS Inc, IBM Corp, Armonk, NY/USA).

\section{Consideraciones Bioéticas}

El estudio fue aprobado por el comité de ética institucional y todos los pacientes incluidos en el estudio brindaron consentimiento informado para su participación.

\section{Resultados}

Los resultados del estudio se resumen en la -Tabla 2.

\section{Tiempo de Isquemia Quirúrgica Promedio}

Los pacientes sometidos a ATR con asistencia robótica, tuvieron una mediana de tiempo de isquemia quirúrgica de 82 minutos (rango 60-120). Se observó una tendencia a un menor tiempo de isquemia a medida que aumentó el número de casos operados en ambos cirujanos que utilizaron isquemia en todas sus cirugías (muestra total: coeficiente $=-0,134$ y $p=0,379$; cirujano 1 : coeficiente $=-0,261$ y $p=0,197$; cirujano 2: coeficiente $=-0,192$ y $p=0,430)$ ( - Figura 1 ).

\section{Días de Hospitalización Promedio}

La mediana de estadía hospitalaria de los pacientes operados fue de 4 días (rango 2-12). La - Figura 2 muestra la distribución de la muestra. Cuarenta y siete pacientes 
Tabla 2 Resumen de los resultados del estudio ( $n=53$ rodillas)

\begin{tabular}{|c|c|}
\hline Categoría & \\
\hline Tiempo Isquemia Mediana (Rango) & $82(60-120)$ \\
\hline $\begin{array}{l}\text { Días Hospitalización } \\
\text { Mediana (Rango) }\end{array}$ & $4(2-12)$ \\
\hline \multicolumn{2}{|l|}{ Dolor Mediana (Rango) } \\
\hline Día 0 & $0(0-10)$ \\
\hline Día 1 & $0(0-6)$ \\
\hline Día 2 & $0(0-6)$ \\
\hline Día 3 & $0(0-5)$ \\
\hline Día Alta & $0(0-4)$ \\
\hline \multicolumn{2}{|c|}{ Uso Opioides (Purga PCA) Mediana (Rango) } \\
\hline Día 0 & $0(0-8)$ \\
\hline Día 1 & $2(0-10)$ \\
\hline Día 2 & $1,5(0-9)$ \\
\hline Día 3 & $0(0-3)$ \\
\hline Día Alta & $0(0-0)$ \\
\hline $\begin{array}{l}\text { Uso por paciente en } \\
\text { Hospitalización }\end{array}$ & $0,3(0-6,5)$ \\
\hline \multicolumn{2}{|l|}{ Tiempo a marcha, $\mathbf{n}(\%)$} \\
\hline Día 1 & $45(84,9)$ \\
\hline Día 2 & $5(9,43)$ \\
\hline Día 3 & $3(5,66)$ \\
\hline \multicolumn{2}{|l|}{ ROM (Ex/Flexº Mediana (Rango) } \\
\hline Día 0 (Por MPC) & $2 / 40(0 / 20-5 / 60)$ \\
\hline Día 1 (Por TMT) & $0 / 45(0 / 30-5 / 120)$ \\
\hline $\begin{array}{l}\text { Control Ambulatorio } \\
{[23 \text { días }(13-50)]}\end{array}$ & $\begin{array}{l}0-97,5 \\
(0 / 60-10 / 120)\end{array}$ \\
\hline \multicolumn{2}{|l|}{ Hematocrito Mediana (Rango) } \\
\hline Preoperatorio & $40,8(30,3-47,7)$ \\
\hline Postoperatorio & $31,7(21,1-38)$ \\
\hline Requerimiento Transfusión, n (\%) & $7(13,5)$ \\
\hline $\begin{array}{l}\text { Eje Mecánico Postoperatorio } \\
\left({ }^{\circ}\right) \text { Mediana (Rango) }\end{array}$ & $179(178,2-180)$ \\
\hline
\end{tabular}

Abreviaturas: Ex, Extensión; Flex, Flexión; MPC, Movilizador Pasivo Continuo; PCA, Anestesia Controlada por el Paciente; ROM, Rango de movilidad articular; TMT, Traumatólogo.

$(88,7 \%)$ permanecieron hospitalizado por menos de 6 días, de los seis pacientes $(11,3 \%)$ restantes, cinco $(9,5 \%)$ estuvieron hospitalizados por 6 días y uno $(1,89 \%)$ por 12 días. En este último paciente de 84 años de edad, se realizó una endoscopía digestiva alta posterior a la cirugía de rodilla con el objetivo de completar el estudio de un antecedente de anemia previo a la ATR. Durante la hospitalización evolucionó con diarrea medicamentosa y profundización de su anemia que requirió transfusiones sanguíneas, explicando la extensión de su estadía hospitalaria.

\section{Dolor Postoperatorio Promedio}

Se observa un bajo dolor (EVA) postoperatorio con una mediana inicial (Día 0) de 0 (rango 0-10) y una mediana previa al alta de 0 (rango $0-4$ ). Se evidenció una disminución progresiva del dolor durante la estadía hospitalaria.

\section{Uso de Opioides en el Postoperatorio}

Durante el día operatorio (Día 0 ) se observa una mediana de 0 purgas diarias (rango 0-8), que evoluciona con un aumento durante el primer día postoperatorio (Día 1) a una mediana de 2 purgas (rango 0-10) y posteriormente se aprecia una disminución progresiva hacia el día del alta, sin purgas anestésicas descritas en los pacientes. Respecto al uso individual de opioides durante la hospitalización, se observa una mediana de 0,3 purgas (rango $0-6,5$ ) por paciente.

\section{Tiempo a Marcha}

La mediana del tiempo a la marcha fue de 1 día (rango 1-3). Cuarenta y cinco pacientes $(84,9 \%)$ iniciaron la marcha durante el primer día postoperatorio.

\section{ROM (Extensión-Flexión ${ }^{\circ}$ Promedio}

Durante el día operatorio (Día 0) se observa una mediana del ROM de $2-40^{\circ}$ (rango 0-20 a 5-60). Al primer día postoperatorio (Día 1) se aprecia una mediana del ROM de $0-45^{\circ}$ (rango 0-30 a 5-120). En el control ambulatorio de los dos primeros meses postoperatorio [23 días (13-50)] existe un aumento de la mediana del ROM a 0-97, $5^{\circ}$ (rango 0-60 a $10-120)$. Veintiún pacientes (40\%) alcanzaron un ROM mayor o igual a $0-90^{\circ}$ y once $(20,7 \%)$ lograron un ROM funcional máximo $\left(0-120^{\circ}\right)$ (-Figura 3).

\section{Pérdida Sanguínea}

Se observa una disminución del hematocrito promedio postoperatorio respecto al preoperatorio, con una mediana inicial de 40,8\% (rango 30,3-47,7) y de 31,1,7\% (rango 21,1-38) posterior a la cirugía. Siete pacientes $(13,2 \%)$ requirieron transfusión de glóbulos rojos [2 unidades $^{2,3}$ ] posterior a la cirugía.

\section{Complicaciones}

Un paciente $(1,89 \%)$ sufrió una dehiscencia del cierre de la artrotomía medial con una luxación patelar subsecuente a las 3 semanas postoperatorias. Fue reintervenido realizándose un cierre del retináculo medial y evolucionó satisfactoriamente. No se presentaron infecciones de herida operatoria. Tampoco se registraron enfermedades tromboembólicas ni infecciones periprotésicas agudas.

\section{Eje Mecánico Postoperatorio}

La mediana final del eje mecánico postoperatorio fue de $179,1^{\circ}$ (rango 178,2-180). La -Figura 4 muestra una evaluación radiológica de un paciente postoperado.

\section{Discusión}

La navegación y los sistemas robóticos en cirugía protésica de rodilla han permitido aumentar la exactitud de los cortes óseos y la precisión del posicionamiento del implante, con un aumento potencial de la seguridad de este procedimiento. ${ }^{11-14} \mathrm{Sin}$ embargo, su impacto clínico ha sido cuestionado. 
140

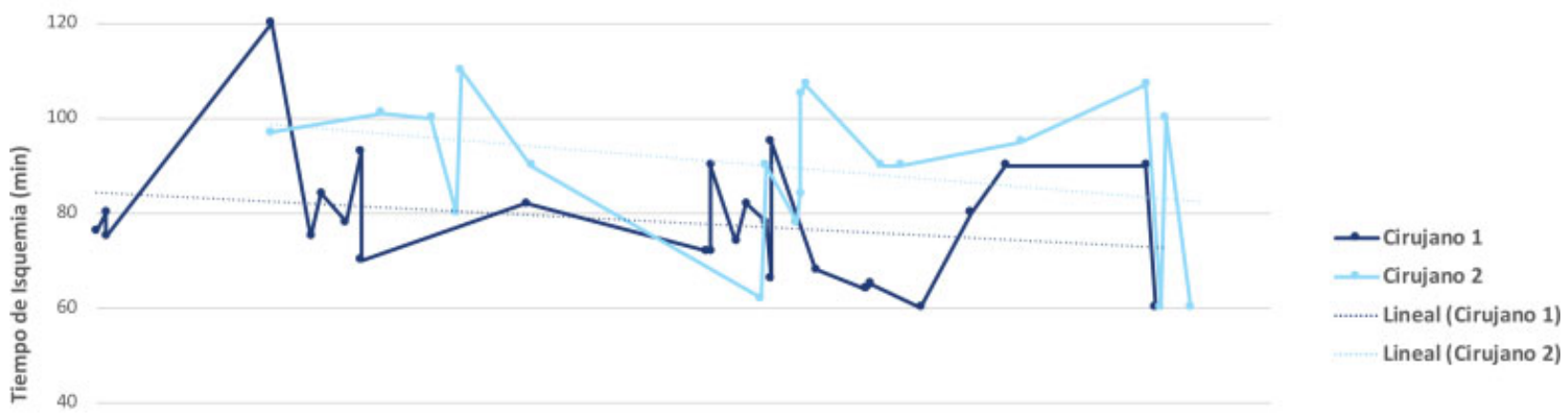

20

22-10-18 21-11-18 21-12-18 20-01-19 $\quad 19-02-19 \quad 21-03-19 \quad 20-04-19 \quad$ 20-05-19

Fig. 1 Distribución de los tiempos de isquemia quirúrgicas. Se muestra la distribución de los tiempos de isquemia de dos cirujanos que utilizaron isquemia en todos sus procedimientos. Se aprecian resultados variables en el tiempo, con una tendencia en ambos cirujanos hacia un menor tiempo de isquemia quirúrgica a medida que cada uno alcanzó más pacientes operados con asistencia de brazo robótico (muestra total: coeficiente $1 / 4-0,134$ y p $1 / 40,379$; cirujano 1 : coeficiente $1 / 4-0,261$ y p $1 / 4$ 0,197; cirujano 2 : coeficiente $1 / 4-0,192$ y p $1 / 40,430$ ). El cirujano 1 operó 27 pacientes $(50,9 \%)$ y el cirujano 2 operó 22 pacientes $(41,5 \%)$.

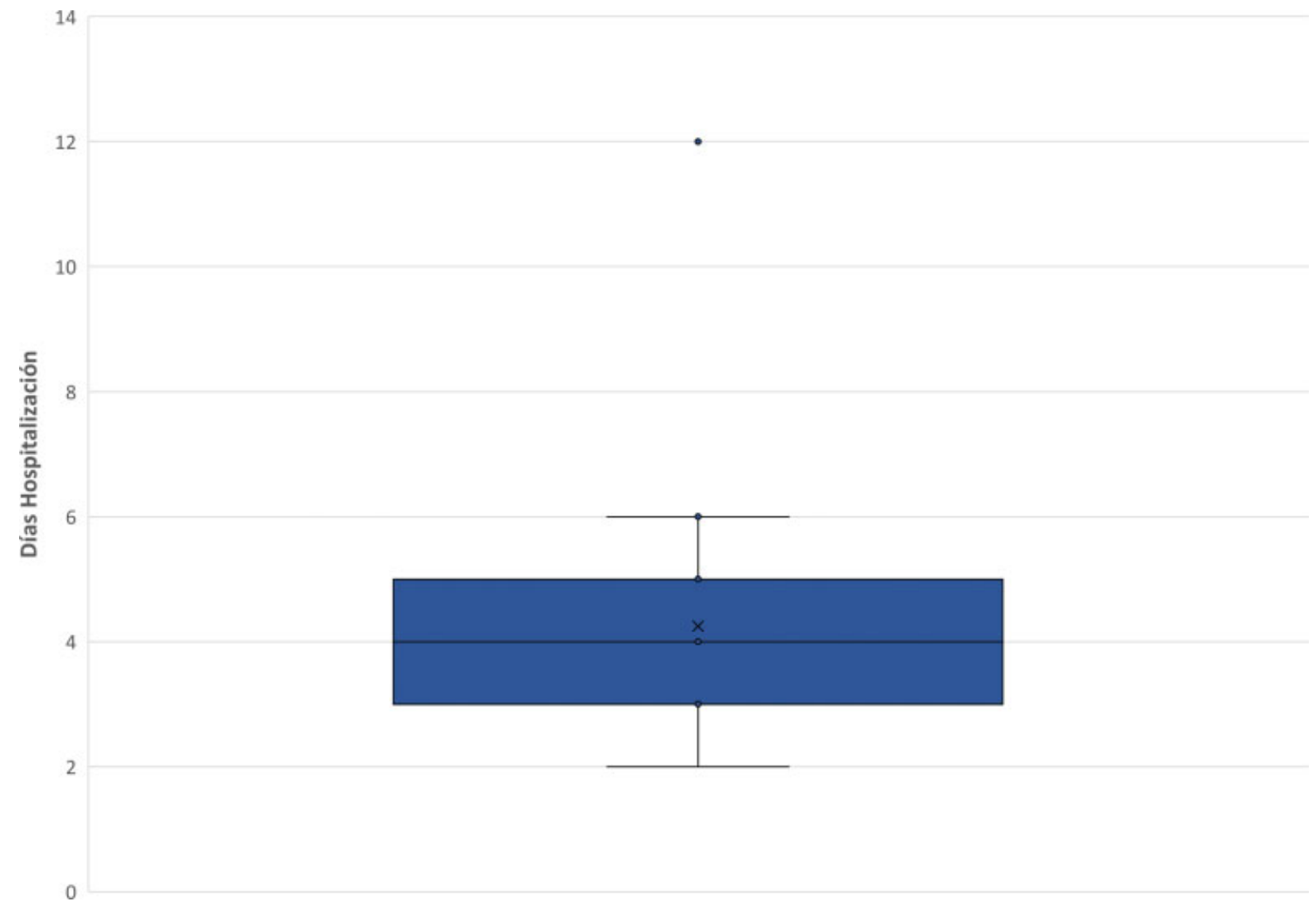

Fig. 2 Estadía Hospitalaria. El gráfico de caja y bigotes muestra la distribución de la estadía hospitalaria en la serie de pacientes estudiados. La media fue de 4,25 1,45 días, la mediana de 4 días y los extremos del diagrama están marcados en 2 y 6 días. Se aprecia que el paciente que permaneció hospitalizado por 12 días se encuentra fuera del diagrama, consideríndose un valor atípico en la muestra. 


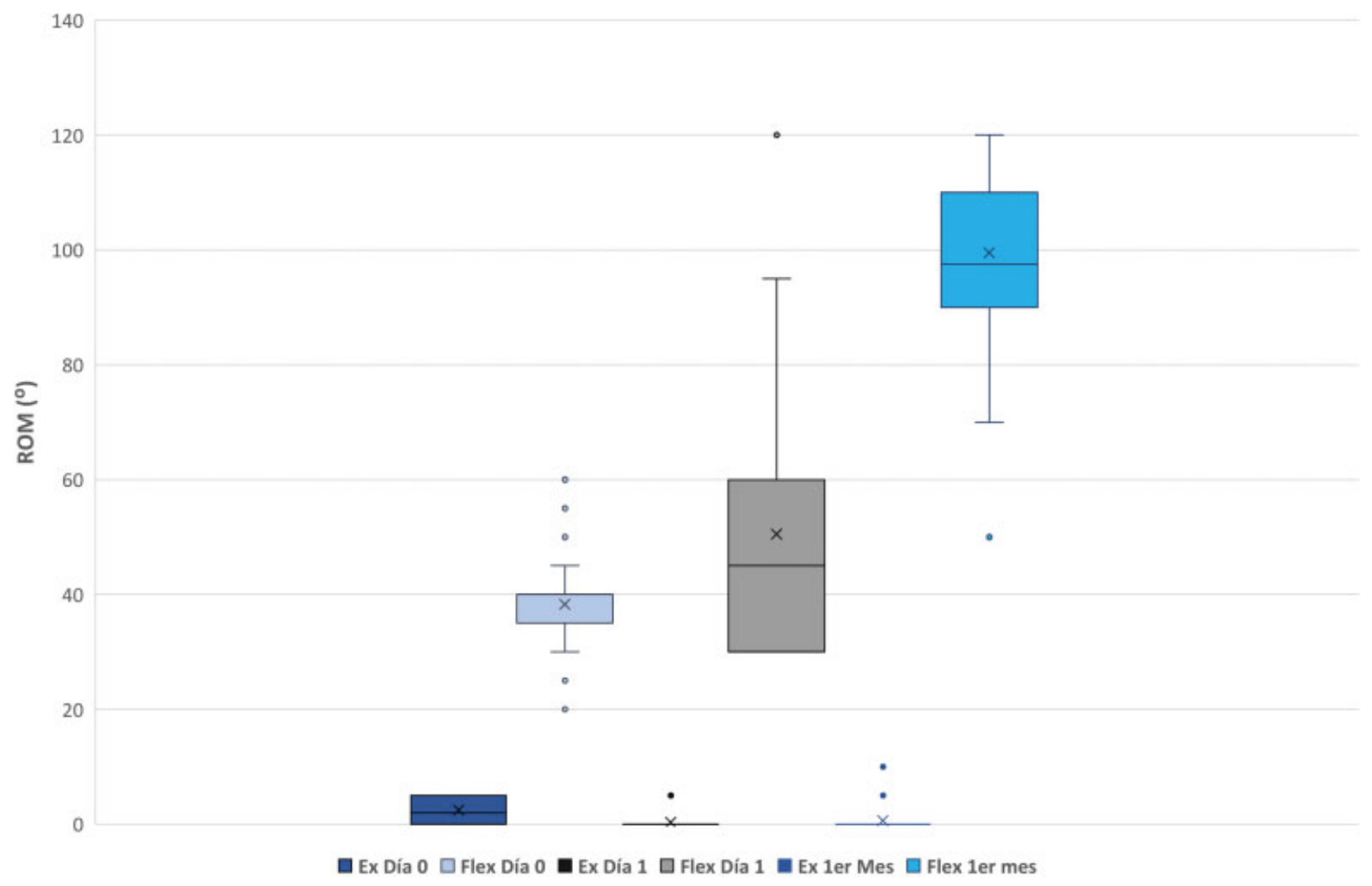

Fig. 3 Distribución del ROM postoperatorio. La figura muestra un aumento progresivo del ROM hacia el primer mes postoperatorio. Un análisis individualizado de paciente evidenció veintiún pacientes (40\%) alcanzaron un ROM mayor o igual a $0-90^{\circ}$ y once (20\%) un ROM funcional máximo $\left(0-120^{\circ}\right)$ al final del seguimiento. Ex: Extensión; Flex: Flexión.

Un estudio reciente de costo-efectividad demostró que la ATR asistida por brazo-robótico representaría un costo significativamente menor que el método convencional, respaldado en una disminución de la tasa de readmisión y menor estadía hospitalaria. ${ }^{15}$ Estudios clínicos comparativos entre los dos métodos han apoyado esa evidencia. ${ }^{11,12,14}$

Los resultados de este estudio describen la experiencia clínica de la primera serie de pacientes operados con asistencia por brazo-robótico en Latinoamérica. Dentro de los valores descritos, destaca una baja percepción del dolor y uso de opioides en el postoperatorio inmediato. Kayani y colaboradores objetivaron que en pacientes sometidos a artroplastia de rodilla unicompartimental, aquellos operados con sistema de brazo-robótico presentaron significativamente menor dolor y uso de opioides en la estadía hospitalaria. ${ }^{18}$ Destaca además que veintiún pacientes (40\%) de la serie logra un ROM mayor o igual a $0-90^{\circ}$ dentro del primer mes postoperatorio. En ese mismo intervalo, once pacientes $(20,7 \%)$ logran rangos funcionales máximos $\left(0-120^{\circ}\right)$. Si bien existe evidencia que reporta una mejoría en la rehabilitación del ROM con los sistemas robóticos, ${ }^{11,14,18}$ esa es dispar y limitada, proviene en parte de estudios antiguos con otros sistemas robóticos, ${ }^{11}$ algunos artículos no cuantifican los rangos, ${ }^{11}$ otros describen la extensión y la flexión por separado ${ }^{11,18}$ o provienen de series de pacientes operados con prótesis unicompartimental, ${ }^{14,18}$ lo que dificulta una comparación exacta con los resultados del presente estudio.
Se ha argumentado que el uso de asistencia robótica puede enlentecer los procedimientos, sobre todo en la fase inicial de aprendizaje y adaptación de uso. ${ }^{19,20}$ Eso expondría a los pacientes a un mayor tiempo quirúrgico con los riesgos asociados, sobre todo en pacientes de mayor fragilidad. En esta serie, que muestra las primeras cirugías consecutivas con asistencia robótica de tres cirujanos, si bien los tiempos de isquemia de los dos cirujanos que utilizaron isquemia en todas sus cirugías fueron variables en el tiempo, se evidenció una tendencia a un menor tiempo de isquemia a medida que aumentó el número de pacientes operados por cirujano. En una revisión de la literatura reciente por Khlopas y col. se describe que no se presentan diferencias significativas en los tiempos quirúrgicos entre las ATR asistidas por brazo-robótico y las ATR por método convencional. ${ }^{12}$ Al igual que en el presente estudio, los autores de la revisión evidenciaron que los tiempos quirúrgicos fueron disminuyendo con el aumento del número de cirugías por cirujano. El valor de corte establecido para diferencias significativas fue de 10-20 cirugías con asistencia robótica por cirujano. ${ }^{12}$ Kayani y col., demostraron una curva de aprendizaje aún menor, posterior a los primeros 7 casos operados por el mismo cirujano, los tiempos quirúrgicos y la ansiedad del equipo quirúrgico fueron significativamente menores. ${ }^{20}$ La mayor parte de la literatura describe el tiempo operatorio total (desde incisión a cierre de la herida operatoria). En el presente estudio, se consideró los tiempos de isquemia (inflado posterior a la colocación de los campos quirúrgicos hasta completada la 


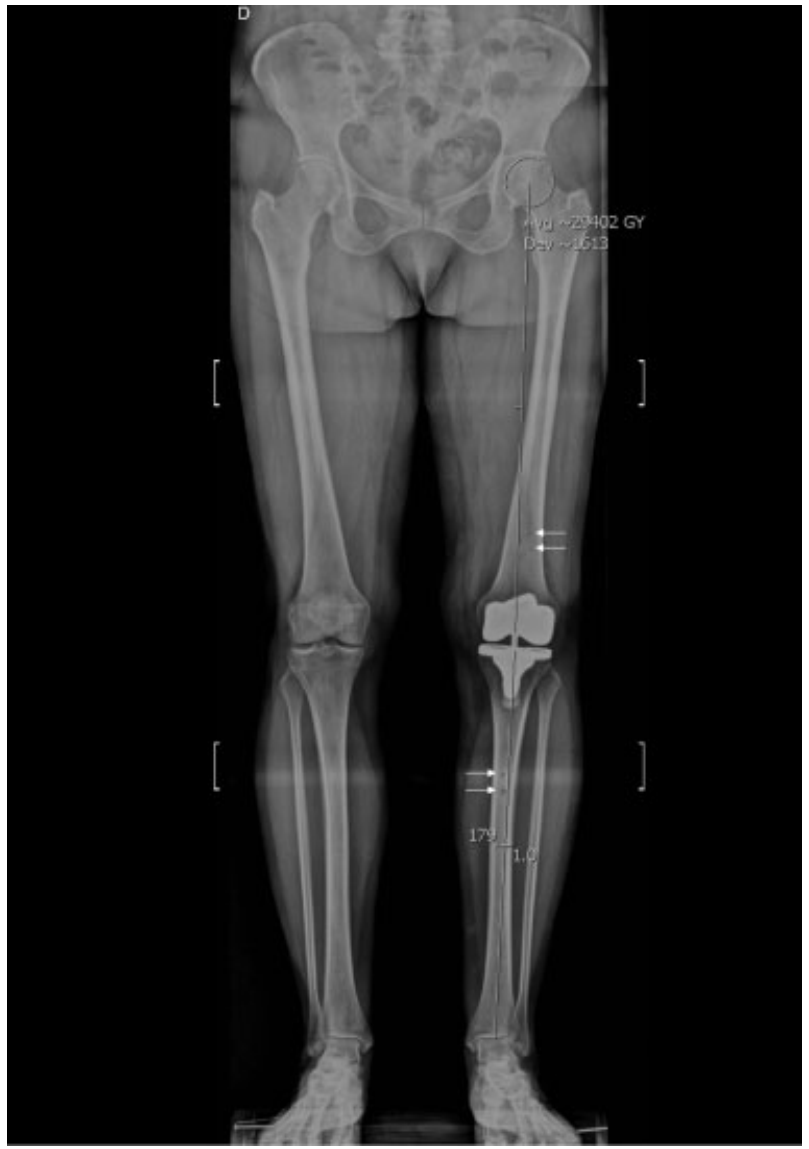

Fig. 4 Evaluación del eje mecánico en un paciente postoperado.

Telemetría de extremidades inferiores de un paciente postoperado. Para la evaluación del eje mecánico de la extremidad inferior, se consideran los ejes de rotación de la cadera, rodilla y tobillo. Se calcula el eje por medio de la diferencia entre el eje mecánico del fémur y la tibia. En este ejemplo el eje mecánico calculado es de $179^{\circ}$. Flechas: muestran los túneles óseos de los trackers utilizados durante la cirugía.

cementación), pues esos sistemas poseen un registro uniforme que no es posible alterar, disminuyendo el riesgo de un sesgo metodológico. Los tiempos totales de cirugía pueden ser ambiguos si no se estandarizan los parámetros. En nuestra práctica clínica, ese dato se considera desde la pausa preoperatoria y es registrado por el personal de pabellón, el que varía según el horario y fecha de la cirugía. Por lo demás, los tiempos totales de cirugía consideran el cierre del abordaje. Ese paso es independiente del uso de asistencia robótica y puede variar según el cirujano responsable.

En la serie reportada, un paciente $(1,89 \%)$ presentó una complicación en relación a la cirugía (dehiscencia del cierre de la artrotomía medial con una luxación patelar subsecuente a las 3 semanas postoperatorias) y siete $(13,2 \%)$ pacientes requirieron una transfusión sanguínea. Lo anterior es compatible con lo reportado en la literatura, donde se describe una baja tasa de complicaciones y efectos adversos y bajos volúmenes de pérdidas sanguíneas. ${ }^{11,12}$

Respecto al eje mecánico postoperatorio, destaca una mediana de $179,1^{\circ}$ con un rango de $178,2-180^{\circ}$. Esos valores concuerdan con la planificación preoperatoria, buscando un eje mecánico de $180^{\circ 13,21}$ y los valores reportados en la literatura. ${ }^{16,17}$ Los resultados sugieren que la ATR con asistencia robótica permitiría obtener resultados postquirúrgicos reproducibles globalmente.

Respecto a las limitaciones del estudio, es importante destacar que es una investigación de carácter descriptiva sin un grupo comparativo, por lo que este primer reporte no permite formular conclusiones comparativas entre la asistencia robótica y el método convencional. Un estudio comparando ambos métodos se encuentra actualmente en ejecución. Además, el seguimiento de las variables analizadas se limita al postoperatorio inmediato y al control ambulatorio del primer mes, pues la experiencia con este nuevo sistema comenzó desde el primer paciente de esta revisión. Aún se desconoce el tiempo al retorno de actividades diarias, la reinserción deportiva, la tasa de complicaciones tardías y la sobrevida del implante. El registro prospectivo de esta serie de pacientes permanece en ejecución para futuros estudios.

\section{Conclusión}

Los resultados clínicos postoperatorios tempranos de esta serie de pacientes operados de ATR con asistencia de brazorobótico, muestran una baja percepción del dolor, con bajo consumo de opioides, una rápida recuperación funcional de la marcha y ROM, y excelentes resultados del punto de vista del eje mecánico postoperatorio.

\section{Conflicto de Intereses}

Lo autor del trabajo declara no tener ningún conflicto de intereses.

\section{Bibliografía}

1 Wallace IJ, Worthington S, Felson DT, et al. Knee osteoarthritis has doubled in prevalence since the mid-20th century. Proc Natl Acad Sci U S A 2017;114(35):9332-9336

2 van den Berg WB. Osteoarthritis year 2010 in review: pathomechanisms. Osteoarthritis Cartilage 2011;19(04): 338-341. Doi: 10.1016/j.joca.2011.01.022

3 Cross M, Smith E, Hoy D, et al. The global burden of hip and knee osteoarthritis: estimates from the global burden of disease 2010 study. Ann Rheum Dis 2014;73(07):1323-1330

4 Inacio MCS, Paxton EW, Graves SE, Namba RS, Nemes S. Projected increase in total knee arthroplasty in the United States - an alternative projection model. Osteoarthr Cartil [Internet] 2017;25 (11):1797-1803. Available from: http://www.ncbi.nlm.nih.gov/ pubmed/28801208

5 Liddle AD, Judge A, Pandit H, Murray DW. Adverse outcomes after total and unicompartmental knee replacement in 101,330 matched patients: a study of data from the National Joint Registry for England and Wales. Lancet 2014;384(9952):1437-1445. Doi: 10.1016/ S0140-6736(14)60419-0 [Internet]

6 Evans JT, Evans JP, Walker RW, Blom AW, Whitehouse MR, Sayers A. How long does a hip replacement last? A systematic review and meta-analysis of case series and national registry reports with more than 15 years of follow-up. Lancet 2019;393(10172):647-654. Doi: 10.1016/S0140-6736(18)32531-5 [Internet]

7 Chang MJ, Lim H, Lee NR, Moon Y-W. Diagnosis, causes and treatments of instability following total knee arthroplasty. Knee Surg Relat Res 2014;26(02):61-67

8 Bini SA, Beneyto FM. Complicaciones de la prótesis total de rodilla. Monografías AAOS-SECOT; 2012 
9 Karthik K, Colegate-Stone T, Dasgupta P, Tavakkolizadeh A, Sinha J. Robotic surgery in trauma and orthopaedics: a systematic review. Bone Joint J 2015;97-B(03):292-299

10 Lang JE, Mannava S, Floyd AJ, et al. Robotic systems in orthopaedic surgery. J Bone Joint Surg Br 2011;93(10):1296-1299http://online. boneandjoint.org.uk/doi/10.1302/0301-620X.93B10.27418 [Internet]

11 Ren Y, Cao S, Wu J, Weng X, Feng B. Efficacy and reliability of active robotic-assisted total knee arthroplasty compared with conventional total knee arthroplasty: a systematic review and meta-analysis. Postgrad Med J 2019;95(1121):125-133

12 Khlopas A, Sodhi N, Sultan AA, Chughtai M, Molloy RM, Mont MA. Robotic Arm-Assisted Total Knee Arthroplasty.J Arthroplasty 2018;33 (07):2002-2006. Doi: 10.1016/j.arth.2018.01.060 [Internet]

13 Mannan A, Vun J, Lodge C, Eyre-Brook A, Jones S. Increased precision of coronal plane outcomes in robotic-assisted total knee arthroplasty: A systematic review and meta-analysis. Surgeon 2018;16(04):237-244. Doi: 10.1016/j.surge.2017.12.003 [Internet]

$14 \mathrm{Fu} \mathrm{J}$, Wang Y, Li X, et al. Robot-assisted vs. conventional unicompartmental knee arthroplasty : Systematic review and meta-analysis. Orthopade 2018;47(12):1009-1017

15 Cool CL, Jacofsky DJ, Seeger KA, Sodhi N, Mont MA. A 90-day episode-of-care cost analysis of robotic-arm assisted total knee arthroplasty. J Comp Eff Res 2019;8(05):327-336
16 Grau L, Lingamfelter M, Ponzio D, et al. Robotic arm assisted total knee arthroplasty workflow optimization, operative times and learning curve. Arthroplast Today 2019;•••:1-6. Doi: 10.1016/j. $\operatorname{artd}$ 2019.04.007 [Internet]

17 Song EK, Seon JK, Yim JH, Netravali NA, Bargar WL. Roboticassisted TKA reduces postoperative alignment outliers and improves gap balance compared to conventional TKA. Clin Orthop Relat Res 2013;471(01):118-126

18 Kayani B, Konan S, Tahmassebi J, Rowan FE, Haddad FS. An assessment of early functional rehabilitation and hospital discharge in conventional versus robotic-arm assisted unicompartmental knee arthroplasty: a prospective cohort study. Bone Joint J 2019;101-B(01):24-33

19 Sodhi N, Khlopas A, Piuzzi NS, et al. The Learning Curve Associated with Robotic Total Knee Arthroplasty. J Knee Surg 2018;31(01):17-21

20 Kayani B, Konan S, Huq SS, Tahmassebi J, Haddad FS. Robotic-arm assisted total knee arthroplasty has a learning curve of seven cases for integration into the surgical workflow but no learning curve effect for accuracy of implant positioning. Knee Surg Sports Traumatol Arthrosc 2019;27(04):1132-1141. Doi: 10.1007| s00167-018-5138-5

21 Donaldson J, Joyner J, Tudor F. Current Controversies of Alignment in Total Knee Replacements. Open Orthop J 2015;9(01):489-494 